\title{
A sustained-release dual-drug ternary salt cocrystal of piperazine ferulate with pyrazinamide: The synthesis, structure and Hirshfeld surface analysis
}

Xue-Zhao Yu ${ }^{1}$, Ling-Yang Wang ${ }^{1}$, Fang Liu ${ }^{1}$, Yan-Tuan $\mathrm{Li}^{1{ }^{12}}{ }^{*}$, Zhi-Yong Wu ${ }^{1}$, and Cui-Wei Yan ${ }^{1, *}$

Supporting Information.

Table S1. HPLC Method Parameters ...................................................................

Figure S1. FTIR spectra of (a) PRZ-FLA, (b) PRA, and (c) PRZ-FLA-PRA. .............1

Figure S2. PXRD results of the undissolved solids after solution experiment. ............2

Figure S3. Dissolution profiles of compressed tablets of PRZ-FLA, PRA and the

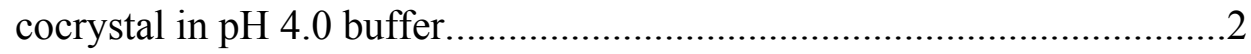

Figure S4. Dissolution profiles of compressed tablets of PRZ-FLA, PRA and the cocrystal in pH 6.8 buffer..................................................................

Figure S5. IDR profiles of PRZ-FLA, PRA and the cocrystal in $\mathrm{pH} 1.2$ buffer............3

Figure S6. IDR profiles of PRZ-FLA, PRA and the cocrystal in $\mathrm{pH} 4.0$ buffer...........4

Figure S7. IDR profiles of PRZ-FLA, PRA and the cocrystal in pH 6.8 buffer...........4 
Table S1. HPLC Method Parameters

\begin{tabular}{|l|l|}
\hline Parameter & Details \\
\hline Column & Diamonsil- $\mathrm{C}_{18}, 5.0 \mu \mathrm{m}, 4.6 \times 150 \mathrm{~mm}$ \\
\hline Mobile phase & $\begin{array}{l}\text { Methanol: ultrapure water: glacial acetic acid (23: 77: } \\
0.3, \mathrm{v} / \mathrm{v} / \mathrm{v})\end{array}$ \\
\hline Column temperature & $30^{\circ} \mathrm{C}$ \\
\hline Sample temperature & $30^{\circ} \mathrm{C}$ \\
\hline$\lambda_{\max }$ & $310 \mathrm{~nm}$ for PRZ-FLA; $268 \mathrm{~nm}$ for PRA \\
\hline Flow rate & $1.0 \mathrm{~mL} \cdot \mathrm{min}^{-1}$ \\
\hline Injection volume & $50 \mu \mathrm{L}$ \\
\hline
\end{tabular}

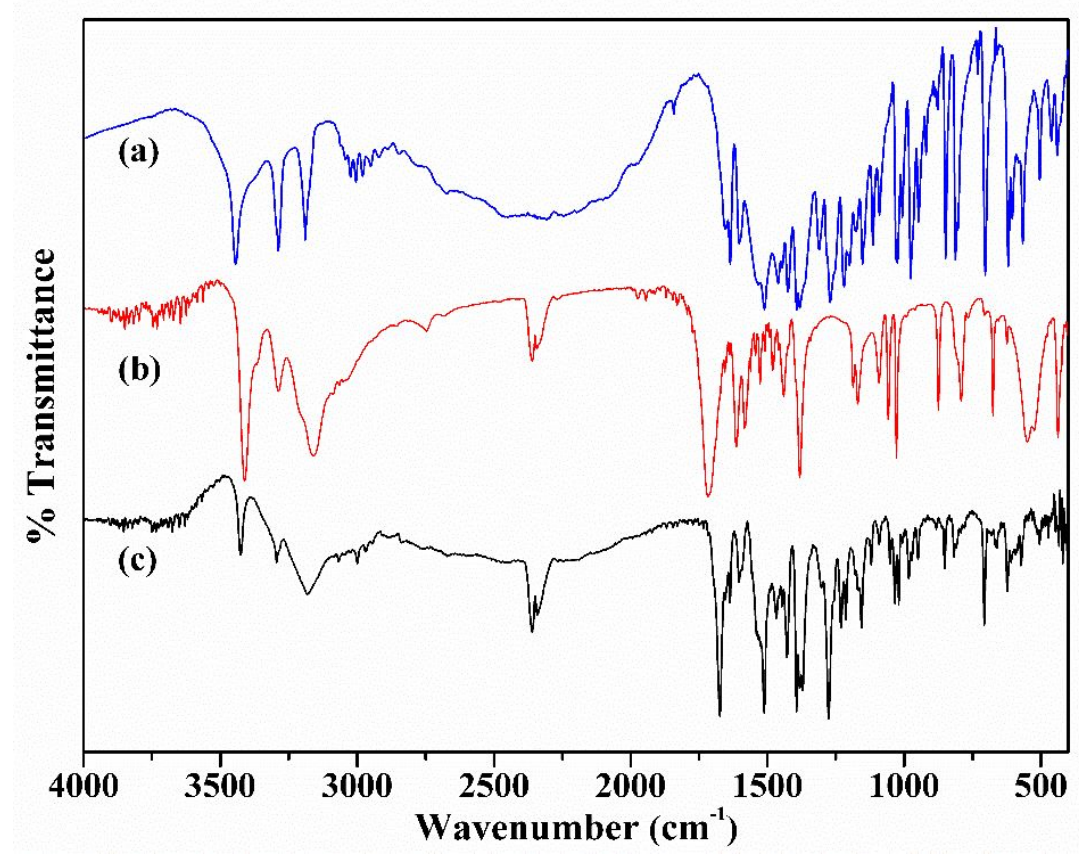

Figure S1. FTIR spectra of (a) PRZ-FLA, (b) PRA, and (c) PRZ-FLA-PRA. 


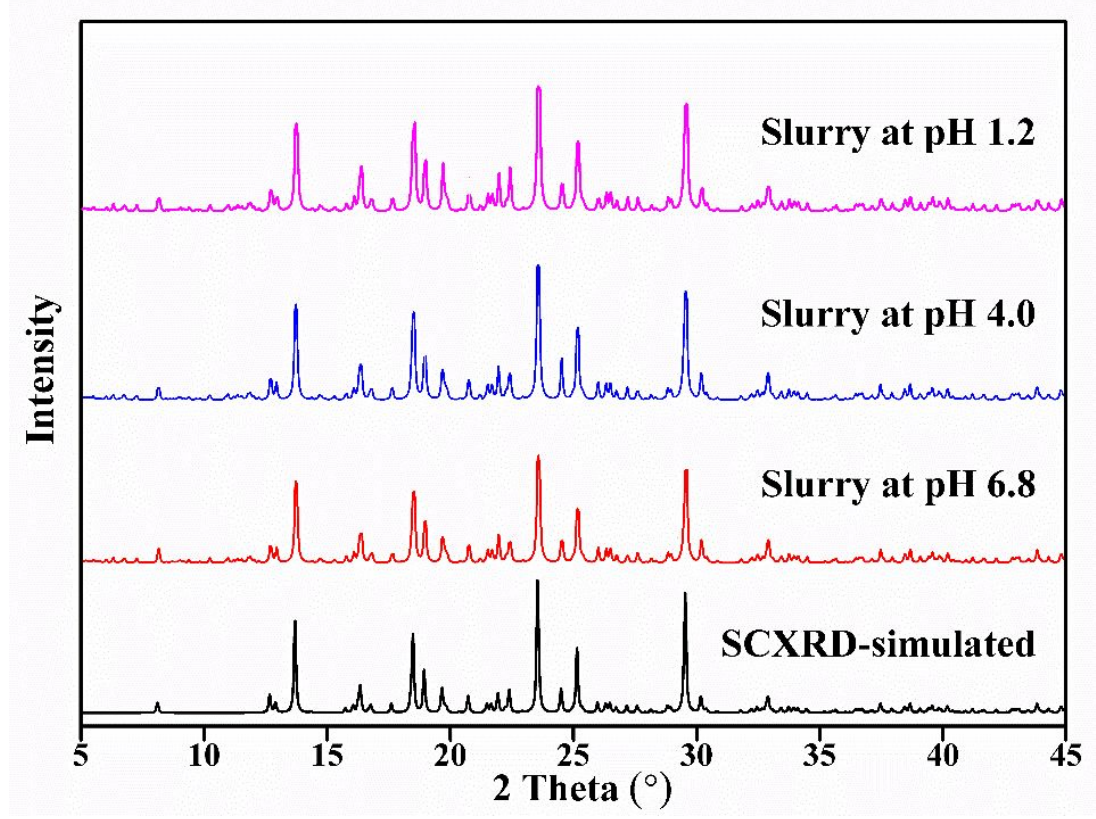

Figure S2. PXRD results of the undissolved solids after solution experiment.

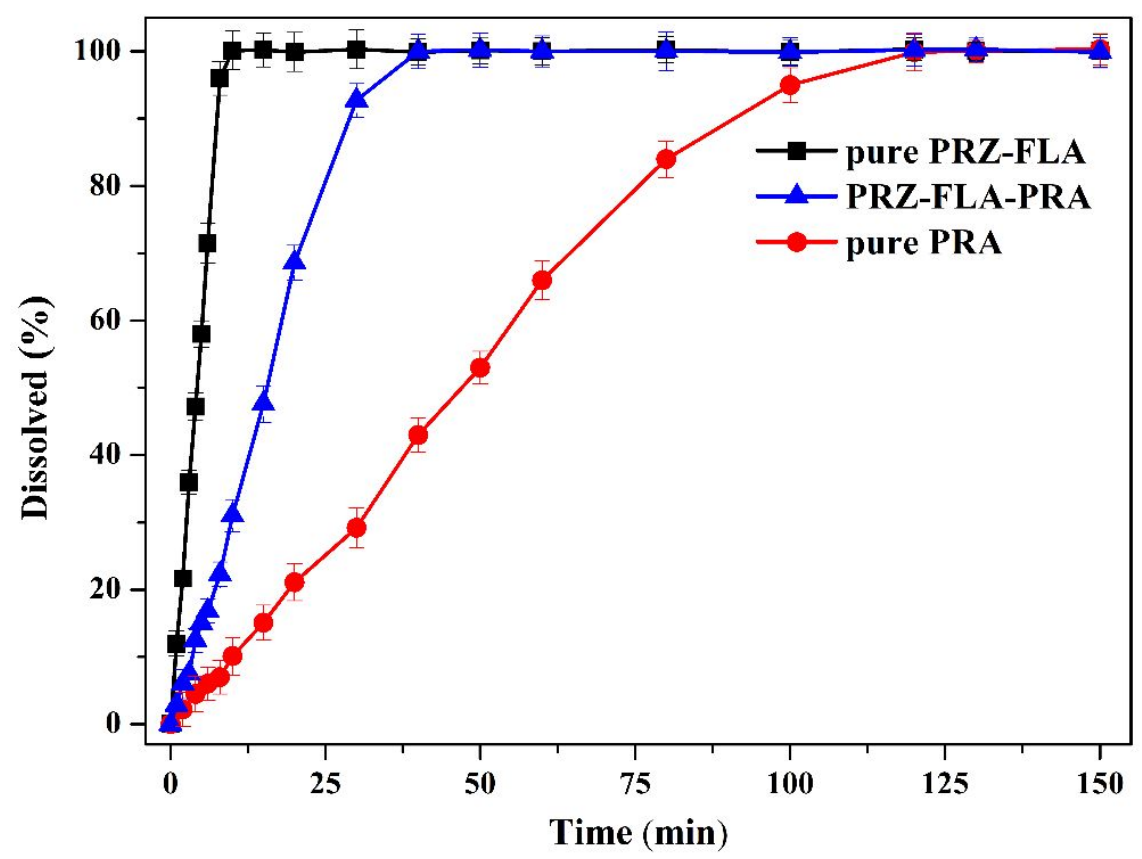

Figure S3. Dissolution profiles of compressed tablets of PRZ-FLA, PRA and the cocrystal in $\mathrm{pH} 4.0$ buffer. 


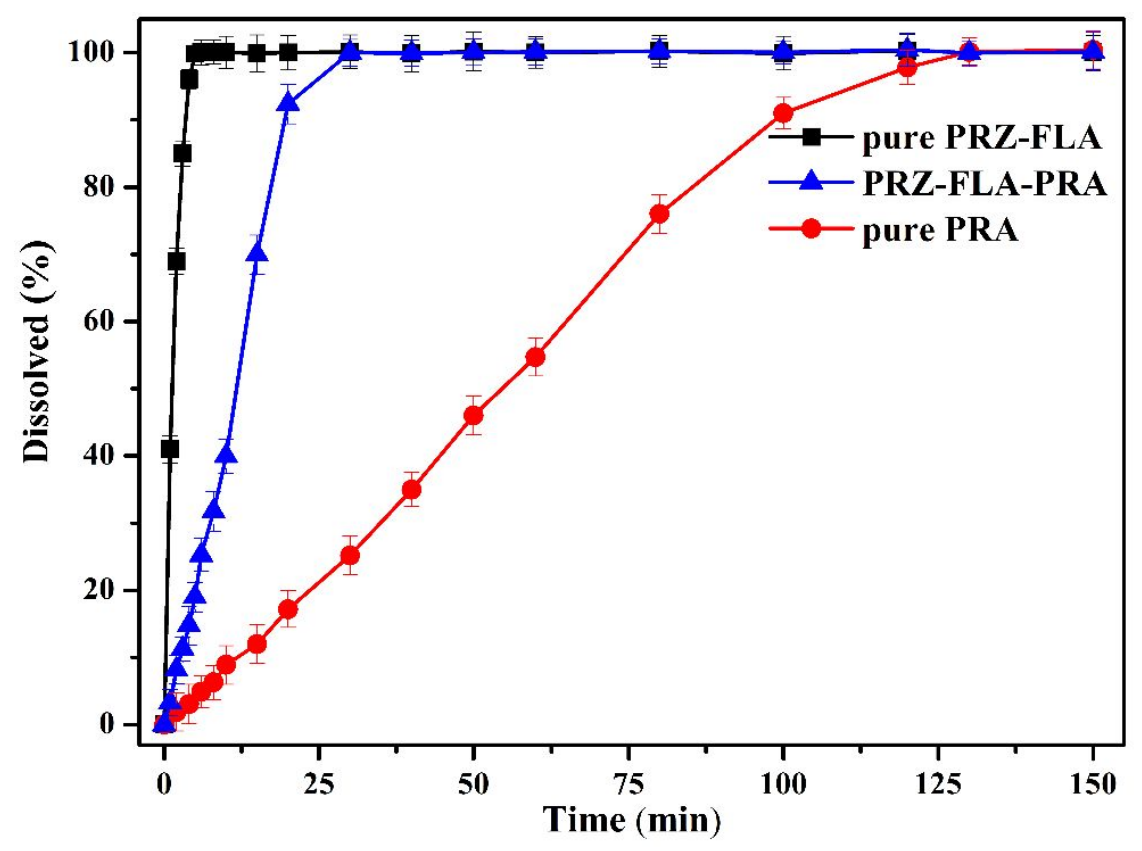

Figure S4. Dissolution profiles of compressed tablets of PRZ-FLA, PRA and the cocrystal in pH 6.8 buffer.

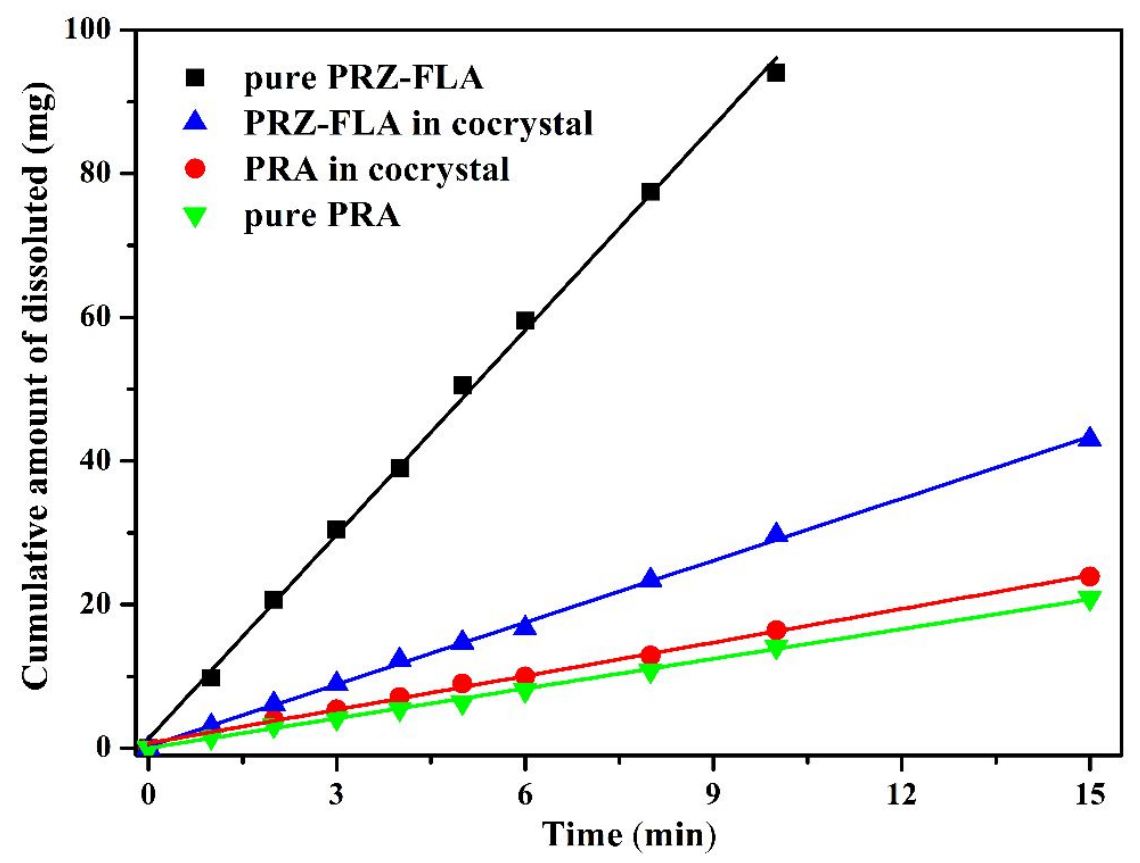

Figure S5. IDR profiles of PRZ-FLA, PRA and the cocrystal in $\mathrm{pH} 1.2$ buffer. 


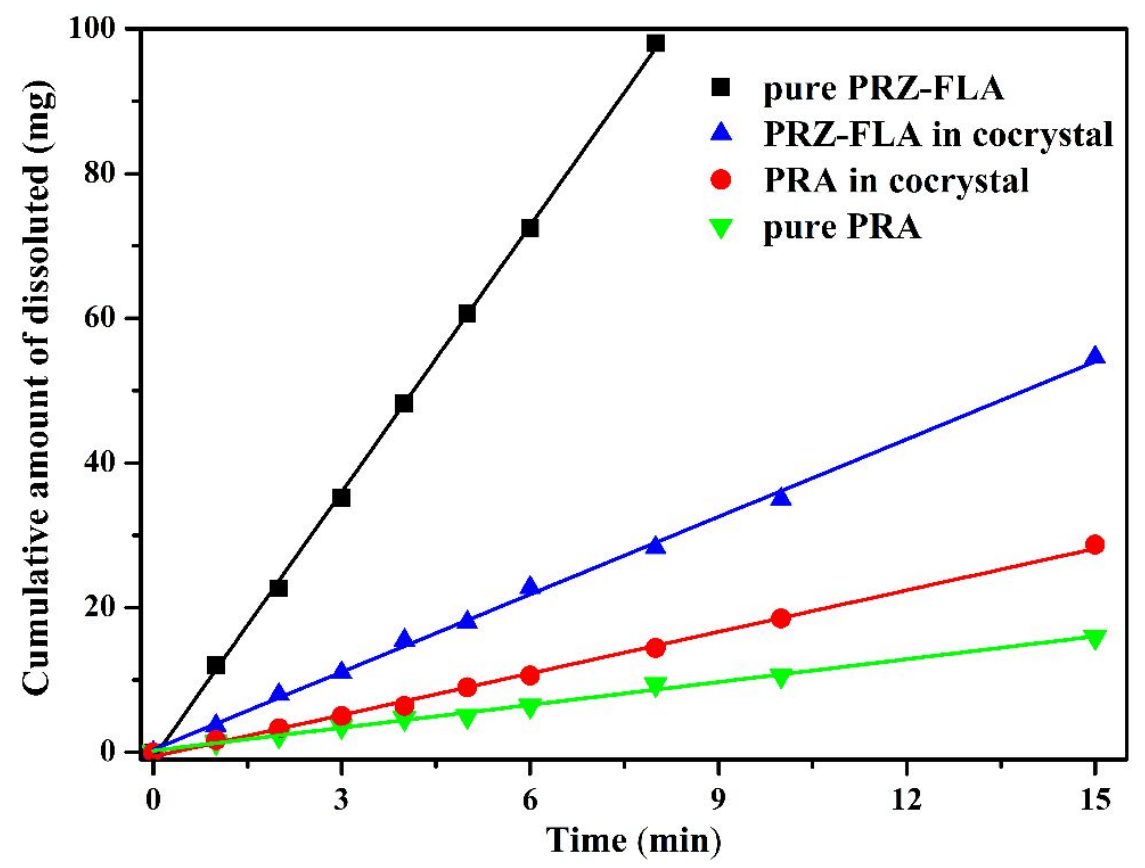

Figure S6. IDR profiles of PRZ-FLA, PRA and the cocrystal in $\mathrm{pH} 4.0$ buffer.

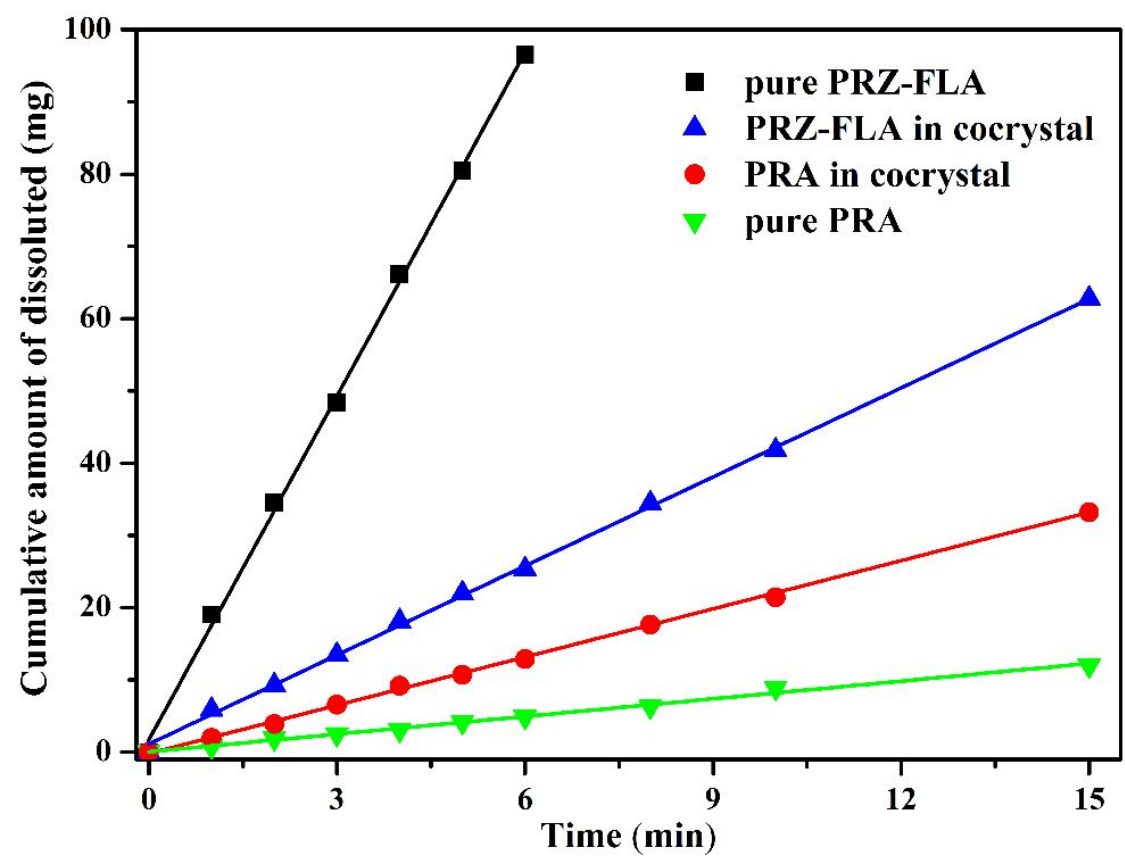

Figure S7. IDR profiles of PRZ-FLA, PRA and the cocrystal in pH 6.8 buffer. 